\title{
Degrees of Doxastic Justification
}

\section{Moritz Schulz ${ }^{1}$}

Received: 23 September 2019 / Accepted: 14 October 2020 / Published online: 30 November 2020 (c) The Author(s) 2020

\begin{abstract}
This paper studies degrees of doxastic justification. Dependency relations among different beliefs are represented in terms of causal models. Doxastic justification, on this picture, is taken to run causally downstream along appropriate causal chains. A theory is offered which accounts for the strength of a derivative belief in terms of (i) the strength of the beliefs on which it is based, and (ii) the epistemic quality of the belief-forming mechanisms involved. It is shown that the structure of degrees of justification converges to ranking theory under ideal conditions.
\end{abstract}

\section{Doxastic Justification}

Justification comes in two flavors: propositional justification and doxastic justification (Audi 1983; Feldman and Conee 1985). To illustrate the difference, consider this case:

Jane and her guru

Jane believes that she is capable of passing the bar exam. Her track record so far is impressive and she has studied hard. Curiously, however, her belief is due to another source of information: her guru told her that she will pass. Needless to say, this guru is notoriously unreliable in his predictions.

There is a sense in which Jane's belief is justified. The evidence she possesses, what she knows about her track record and the amount of studying she has done, sufficiently supports the proposition she believes. But there is also a sense in which Jane's belief is not justified, for her belief is not adequately based on the evidence: she believes what she believes for the wrong reasons. These two senses are standardly marked by saying that Jane's belief is propositionally justified but lacks doxastic justification.

Just like propositional justification, doxastic justification can be more or less strong. For instance, in one variation of the case above, Jane may believe that she is capable of passing the exam because her track record is impressive and she has studied hard. Compare this with a case in which Jane's supervisor additionally told her that she is confident that Jane will pass, and Jane is sensitive to this additional reason. It seems

\footnotetext{
Moritz Schulz

moritz.schulz@tu-dresden.de

1 TU Dresden, Dresden, Germany
} 
that in the second case, Jane's doxastic justification is stronger, or, to put it differently, her degree of doxastic justification is higher. ${ }^{1}$ As a first pass, the more (independent) reasons adequately ground one's belief, and the stronger these reasons are, the higher one's degree of doxastic justification.

What constitutes a certain degree of doxastic justification? What principles could one expect degrees of doxastic justification to obey? And what distinguishes degrees of imperfect thinkers from degrees of perfect beings?

Somewhat surprisingly, degrees of doxastic justification are largely underresearched. There is considerable work on the nature of doxastic justification. And there is, of course, a longstanding debate on degrees of propositional justification (not always under this name: just think, for example, of the literature on rational degrees of belief). This paper is an initial attempt to provide a (formal) framework for studying degrees of doxastic justification.

Why do degrees of doxastic justification matter? One reason is that degrees of doxastic justification can be expected to advance our understanding of the link between belief and rational action. It is widely held that whether one should rely on a certain proposition in one's practical reasoning partly depends on what is at stake (Cohen 1999; Gerken 2011; Fantl and McGrath 2002; Stanley 2005). ${ }^{2}$ Details may vary, but in some way or other many would like to be able to say that the higher the stakes, the higher the epistemic standard a belief must satisfy for being appropriately relied upon in practical reasoning. Now, it is a plausible thought that epistemic standards are identical to, or at least closely connected to, constraints on one's degrees of doxastic justification. This would put the following constraint in place: the higher the stakes, the higher one's degree of doxastic justification should be for a belief to be appropriately relied upon in practical reasoning.

The interaction between belief and rational action is often discussed in the context of possible stake-effects on knowledge. For instance, contextualists hold that high stakes require higher epistemic standards for a knowledge-ascription to be true in a given context (Cohen 1999). Sensitive invariantists, in contrast, claim that the epistemic standard required by knowledge can itself be affected by the practical situation of the agent (Fantl and McGrath 2002; Stanley 2005). On the proposed identification of epistemic standards with degrees of doxastic justification, stake effect on knowledge could be redescribed as effects on the degree of doxastic justification required by knowledge (cf. Fantl and McGrath 2002: 77).

Tying degrees of doxastic justification closely to epistemic standards imposes a constraint on one's theory of doxastic justification. According to this constraint, it is permissible to rely on a proposition $p$ in practical reasoning when the stakes are high/low only if one's degree of doxastic justification for $p$ is high/low. This general constraint can be expected to have further consequences down the way. A particularly

\footnotetext{
1 Taking doxastic justification to come in degrees does not exclude a notion of doxastic justification simpliciter. The latter may correspond, for instance, to a sufficiently high degree of doxastic justification (cf. Spohn 2012: 482). See also p. 31 for more discussion.

2 Williamson (2000: 99) suggests that outright beliefs come in degrees, varying with how much one is willing to rely on a belief in one's high and low stakes decision-making. On this suggestion, it is a natural expectation that having a belief of a certain degree is rational only if the belief is doxastically justified to the very same degree.
} 
important question is how it bears on the issue of closure. If degrees of doxastic justification are closely related to knowledge, for instance by providing variable standards one's beliefs must satisfy in relation to the practical stakes, then a plausible closure of knowledge under competent deduction requires, or at least strongly suggests, that degrees of justification are equally apt for closure. ${ }^{3}$ When two or more premises are doxastically justified to a certain degree or higher, a logical consequence should be justified to the same degree or higher, provided it has been properly deduced.

Even without a direct link between degrees of doxastic justification and knowledge, the general role degrees of doxastic justification are supposed to play for proper action suggests closure under competent deduction. As various authors argue, relying on a proposition means to exclude all possibilities which are relevant in light of the stakes the agent faces (Hawthorne and Stanley 2008: 572; Weatherson 2012). Yet if all possibilities on which any single proposition would be false are excluded, then all possibilities on which a logical consequence of these premises would be false will be excluded as well. Thus, if reliance comes with exclusion of error within a certain range of possibilities determined by the stakes, closure emerges as a side constraint. In addition, there are more formal arguments suggesting that the set of premises one should rely on in practical deliberation is closed under competent deduction (Fantl and McGrath 2002, App. II). Although there is clearly a debate to be had about a closure-constraint on degrees of doxastic justification, I will take this constraint on board for the purposes of this paper.

Degrees of doxastic justification are also likely to matter for a theory of rationality along other dimensions. It is an increasingly popular thought that rationality is closely linked to reason-responsiveness (cf. Kiesewetter 2017; Lord 2018). On this approach, and modulo some further modifications, an attitude is rational iff it is an appropriate reaction to the reasons the agent has. But this requires an account of how appropriate reactions to one's reasons look like. Degrees of doxastic justification can serve here as a case study by zooming in on the epistemic attitude of belief. Finally, degrees of doxastic justification could play a role for rational belief revision. More specifically, some authors have observed that it might matter for belief revision which beliefs are basic and which derivative (cf. Hansson 1999; Rott 2000). Add to this that it is clearly relevant to belief revision to what degree one's beliefs are justified and it is only a small step to conjecture that degrees of doxastic justification could shed some light on rational belief revision as well.

Of course, these considerations are just a first touch on the role degrees of doxastic justification could play in an overall theory of rationality. But they provide, I hope, initial motivation to think that degrees of doxastic justification are important.

As doxastic justification hinges on dependency relations among beliefs, a framework for doxastic justification requires a way of representing how beliefs can depend on other beliefs. It is one contention of the present paper that so-called causal models can do a good job in this regard, for they provide a precise way of tracking causal dependencies. This may be surprising at first as causal models are typically not applied

\footnotetext{
3 For a defense of a closure-condition on knowledge, see e.g. Hawthorne (2005), Pritchard (2005: 167f.), Sosa (1999) and Williamson (2000: 117).
} 
to epistemic states like belief states. But as we shall see, causal models do well in representing how the combination of various beliefs generates new beliefs.

The paper is structured as follows. It starts by relating doxastic justification to causal models (Sects. 2-3). Next, two constraints on the logic of degrees of doxastic justification are defended (Sect. 4). Section 5 then presents a theory of degrees of doxastic justification designed to meet these constraints. Section 6 applies the theory to perfect thinkers. Section 7 concludes, followed by a short "Appendix".

\section{Causal Models for Belief}

Doxastic justification is at least partly a matter of dependencies among beliefs. As a working hypothesis, I assume that such dependencies are to be construed in terms of causation by the right kind of causes in the right kind of way (cf. Korez 1997). Let me point out, though, that a causal interpretation of the model to be presented below is optional: the dependency relation constituting the core aspect of doxastic justification may well be understood in non-causal or not-merely-causal terms. Moreover, the account is general enough to cover dispositional aspects of doxastic justification by being sensitive not only to what actually caused a given belief but also to what would have caused the belief under different circumstances. Given that the account primarily targets the structure of doxastic justification, it is compatible with a wide variety of views on the nature of doxastic justification. ${ }^{4}$

That being said, I introduce in this section a framework for representing the causal relations among a given agent's beliefs. I do so by facilitating so-called causal models. ${ }^{5}$ A causal model of an agent's beliefs describes the causal structure these beliefs have, no matter whether the causal processes are good or bad in an epistemic sense. That is, causal models represent in a purely descriptive fashion how the beliefs of a given agent depend on other more basic beliefs. In this way, these models capture how an agent responds to various combinations of other beliefs she has. ${ }^{6}$

Let us introduce the following definition of a causal model, which is due to Halpern and Hitchcock (2010):

Definition 2.1 (Causal Model) A causal model $\mathcal{M}$ is a pair $(\mathcal{S}, \mathcal{F})$, in which $\mathcal{S}$ is a signature and $\mathcal{F}$ is a set of structural equations. A signature is itself a tuple $(\mathcal{U}, \mathcal{V}, \mathcal{R}) . \mathcal{U}$ and $\mathcal{V}$ are disjoint sets of variables, while $\mathcal{R}$ specifies the possible range of these variables. $\mathcal{U}$ contains the exogenous variables whereas $\mathcal{V}$ contains the endogenous variables. ${ }^{7}$

\footnotetext{
${ }^{4}$ See Neta (2019) for a helpful discussion of dispositionalist, representationalist and hybrid accounts of the nature of doxastic justification.

5 Here I am indebted to McCain's (2012) account of the basing relation in terms of an interventionist theory of causation. The theory of causal models is most prominently developed in Pearl (2000). My presentation closely follows Halpern and Hitchcock (2010).

${ }^{6}$ As far as I can see, no substantive assumptions about the nature of belief have to be made, as long as it is granted that we have some beliefs because of others. For instance, if having a belief consists in having a certain complex disposition, then all that has to be assumed is that a disposition can be formed in response to other dispositions one already has.

${ }^{7}$ For reasons of simplicity, I assume that the two sets are finite.
} 
Causal models can be used to describe the causal dependencies of a given agent's belief at a given time. In order to do so, let us interpret the variables of a causal model as propositions $p, q, r, \ldots$. They are taken to specify those propositions which the agent may or may not believe. These propositions can be complex, but there is no need to assume that they form a complete algebra (this will only be assumed for perfect thinkers; see Sect. 6). Moreover, logically equivalent propositions are allowed to be distinct. Propositions in this model are intended to cover the range of what is within the agent's cognitive reach (cf. the notion of epistemic reach in Egan 2007: 8). A proposition is assumed to be within the cognitive reach of an agent if the agent is capable of entertaining the proposition, independently of whether the agent has actually entertained it.

The exogenous variables correspond to the basic beliefs of the agent, that is, beliefs which are held independently of other beliefs. Endogenous variables correspond to propositions which are believed (or not believed) because of other beliefs of the agent. They correspond to the agent's derivative beliefs. The choice of variables makes it clear that the scope of the model is somewhat limited: it makes no attempt to explain how basic beliefs are formed in response to perceptual states. ${ }^{8}$

The range $\mathcal{R}$ of the variables will be assumed to be binary, containing just the two values $\mathbf{b}=$ 'is believed' and $\mathbf{n b}=$ 'is not believed'. So, if a proposition is assigned the value $\mathbf{b}$, this means that the proposition is believed by the agent. If assigned $\mathbf{n b}$, then this is not the case. For all that the value nb says, the agent may either be agnostic about the proposition or believe its negation. The binary range of variables also indicates that the model is designed to deal with qualitative (or outright or full) belief rather than with credences.

The set $\mathcal{F}$ of structural equations associates every endogenous variable $v$ with a function $F_{v}\left(x_{1}, \ldots, x_{n}\right)$ which takes as input values $x_{1}, \ldots, x_{n}$ of other variables, including the exogenous variables. Formally, $F_{v}$ operates on $n$-tuples of values in $\mathcal{R}$, where each position in the tuple is associated with a variable in $(\mathcal{U} \cup \mathcal{V}) \backslash\{v\}$. The output of the function $F_{v}$ is a value for $v$ given values of certain other variables as input.

The basic thought is that the structural equations capture how a belief (or absence thereof) depends on the presence (or absence) of other beliefs. As an example, consider a conjunction, $p \wedge q .{ }^{9}$ Sally might believe that Ben and Mary are in town, $p \wedge q$, if and only if she believes that Ben is in town, $p$, and she believes that Mary is in town, $q$. That is, her belief in the conjunction depends on her beliefs in the two conjuncts. The exact workings of this dependency may be brought out this way: ${ }^{10}$

$$
p \wedge q=\left\{\begin{array}{l}
\mathbf{b} \text { if } p=\mathbf{b} \text { and } q=\mathbf{b} \\
\mathbf{n b} \text { otherwise }
\end{array}\right.
$$

\footnotetext{
8 But see McCain (2012) for how this could be done.

9 For better readability, I omit quotation marks and corner quotes when referring to complex formulas.

10 Expressions like like ' $p=\mathbf{b}$ ' are shorthand for 'The value of the proposition $p$ is the value b' (cf. Halpern and Hitchcock 2010).
} 
Fig. 1 Not believing a conjunction

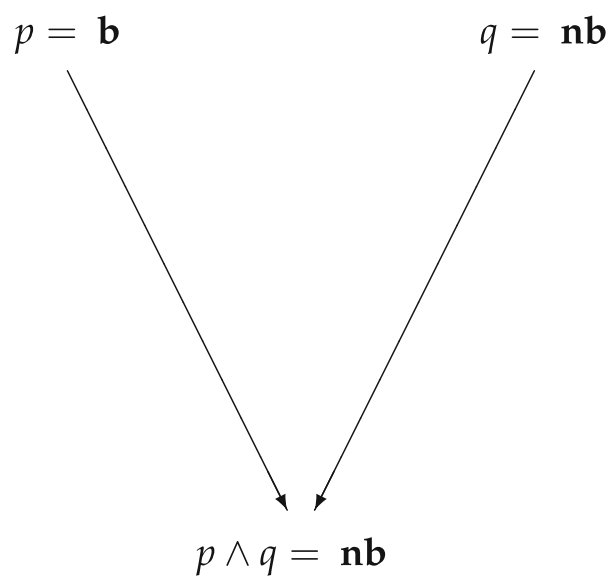

The equation describes how the value of a propositional variable, in our case a conjunction, can be computed out of the values of its conjuncts. ${ }^{11}$ (The variables figuring in an equation of another variable are called its parents.) For further illustration, see Fig. 1: a conjunction is not believed because one of its conjuncts is not believed.

The set of structural equations can be pictured to come in layers. On a first layer, structural equations take exogenous variable as arguments and assign a value to an endogenous variable, typically a more complex formula constructed out of the input variables. These equations represent how, on a first layer, beliefs are derived from basic beliefs. On higher layers, structural equations also take endogenous variables as arguments to assign values to other, typically even more complex, endogenous variables. For example, a structural equation can take a derivatively believed conjunction, $p \wedge q$, and a basic belief $r$ as arguments to assign a value to $(p \wedge q) \vee r$. Formally, this function has just two arguments, a conjunction and an atomic proposition (see also Fig. 7 on p. 28). Therefore, this function differs from a function which accepts the three atomic propositions $p, q$ and $r$ as arguments and directly assigns a value to $(p \wedge q) \vee r$, without requiring a value for $p \wedge q$ first. Which of these two functions should occur in a model depends on the causal links instantiated by the agent's reasoning one wishes to describe. There may be fast reasoners which directly infer $(p \wedge q) \vee r$ from the belief base. More circumspect reasoners might instead first infer the conjunction before they tackle the more complex disjunction.

Structural equations only assign values to the endogenous variables. What about the exogenous variables in $\mathcal{U}$ ?

Definition 2.2 (Context) A context is a function which supplies each exogenous variable with a value in $\mathcal{R} .^{12}$

\footnotetext{
11 The present equation instantiates closure under conjunction for belief. But it serves here a merely illustrative function. It is compatible with a causal model where not all or even none of the agent's beliefs are closed under conjunction. In such a case, the present equation would simply not be part of how the dependencies of an agent's belief system are to be captured.

12 See Halpern and Hitchcock (2010).
} 
Contexts supply values for the exogenous variables from outside the model, indicating, on our interpretation, that basic beliefs are beliefs held independently of other beliefs.

I assume that the structural equations combined with a context determine, in a stepwise fashion starting with the base beliefs, a unique value for each propositional variable in the model. ${ }^{13}$ Ultimately, then, a causal model in a given context determines a unique function which assigns every variable (aka proposition) in the model either the value b (for 'is believed') or nb (for 'is not believed'). To wrap things up, consider then the following definition:

Definition 2.3 (Causal Model for Belief) A causal model for belief is a pair consisting of a causal model with a binary range $(\{\mathbf{b}, \mathbf{n b}\})$ and a context for this model.

Let me add that these models have an intended interpretation as outlined in this section. They can be non-ideal and may even reflect flaws in the reasoning of a particular agent.

\section{Assigning Justification}

A natural picture about doxastic justification takes base beliefs to provide the ultimate grounds for all other beliefs. If all goes well, base beliefs pass their justification upward along valid causal chains. If something goes wrong, only part or none of the justification gets transmitted. ${ }^{14}$

Base beliefs are represented in our model by the exogenous variables. Structural equations describe the belief-forming mechanism by which a derivative belief is produced. The degree of justification a derivative belief enjoys depends on both the strength of its ultimate reasons, i.e. the base beliefs, and the epistemic quality of the belief-forming mechanism. It is therefore a natural starting point to supply the exogenous variables and the structural equations with additional values representing the degree of justification of the base beliefs and the degree of strength of the beliefforming mechanisms. This thought is reflected in the following definition:

Definition 3.1 (Justification Function) A function $J$ into the set $\mathbb{N}_{0} \cup\{\infty\}$ of the natural numbers including zero and infinity is said to be a justification function if

(i) its domain is the union $\mathcal{U} \cup \mathcal{F}$ of the exogenous variables and the structural equations of a given causal model for belief, and

(ii) for all exogenous variables $v$ which are assigned the value $\mathbf{n b}$ in the model, $J(v)=0 .{ }^{15}$

The second clause amounts to saying that in the present model only beliefs can be doxastically justified. It should be admitted that this is a limitation of the account of

\footnotetext{
13 In the terminology of causal models, it is assumed that the model is acyclic (cf. Halpern and Hitchcock 2010). In particular, this means that circular causal chains are excluded.

14 I have to set aside the larger debate about foundationalism (Alston 1976) versus coherentism (Bonjour 1976). Although the current picture is foundationalist in spirit, it should be obvious that it does not require base beliefs to be absolutely certain or unrevisable. For a recent defense of coherentism in a graph-theoretic framework close to the present one, see Berker (2015).

15 It would be reasonable but not strictly necessary to add that the set of justified base beliefs must be consistent. See the discussion of Correctness in Sect. 6 for more on consistency.
} 
doxastic justification developed in this paper. There are other epistemic attitudes such as suspension of judgment (and possibly disbelief) which can be doxastically justified (Feldman and Conee 1985).

Adding a justification function to a causal models brings us to the following definition:

Definition 3.2 (Causal Model for Doxastic Justification) A pair consisting of a causal model for belief and a justification function is called a causal model for doxastic justification. $^{16}$

To get a better picture, let us start by looking at the base beliefs. In a causal model for doxastic justification, a justification function provides base beliefs with degrees of justification. The interpretation of assigned values is the obvious one: 0 means that the proposition is not justified, while positive numbers mean that it is justified to some degree, and the more so, the higher the number. Including $\infty$ as a potential value opens up the possibility of a maximal degree of justification which could be instantiated by perfect thinkers (see Sect. 6).

The choice of a discrete order is partly due to considerations of simplicity and partly owes itself to the fact that the justification of what will be called the "sum rule" in Sect. 4 requires the natural numbers as an underlying domain. But degrees of justification can be made continuous and defined over the real numbers (cf. Spohn 2012: ch. 5). This would capture the intuitively plausible thought that there are no "jumps" in the strength of justification.

A causal model for doxastic justification provides a base variable with two values: a value signaling whether the proposition is believed and a value representing its degree of justification. Figure 2 illustrates how a disjunction may be believed to degree 2 based on one disjunct being believed to the same degree, while the other disjunct is not believed at all. Presupposed in this example is a structural equation for disjunction defined in the following way:

$$
p \vee q=\left\{\begin{array}{l}
\mathbf{b} \text { if } p=\mathbf{b} \text { or } q=\mathbf{b} \\
\mathbf{n b} \text { otherwise. }
\end{array}\right.
$$

The cases presented so far could make it seem as if structural equations representing belief-forming mechanisms closely follow the truth tables for the truth-functional connectives. Although this will often be so, the correspondence is far from universal. For one, belief-forming mechanisms can in some cases be defective. For instance, a conjunction may be treated like a disjunction, so that a conjunction is inferred from just one of its conjuncts (resulting in an unjustified belief). But even a flawless belief need not necessarily be formed in analogy to a corresponding truth table. The reason for this is that although the value b for belief behaves very much like the truth-value 'true', the value $\mathbf{n b}$ for non-belief behaves very unlike the classical truth-value 'false'. ${ }^{17}$ To

\footnotetext{
16 Let me mention one issue only to set it aside. Given that causal models are assumed to be acyclic (fn. 13), doxastic justification cannot be circular. This may be a virtue, but it could also be a vice in light of cases that potentially instantiate circular chains of doxastic justification. For discussion, see McCain (2012: 4.3). 17 It is true, though, that in the equation above $\mathbf{n b}$ behaves very much like a non-classical truth-value "not true" on some ways of construing it (compare e.g. the Kleene tables for disjunction).
} 
Fig. 2 Believing a disjunction

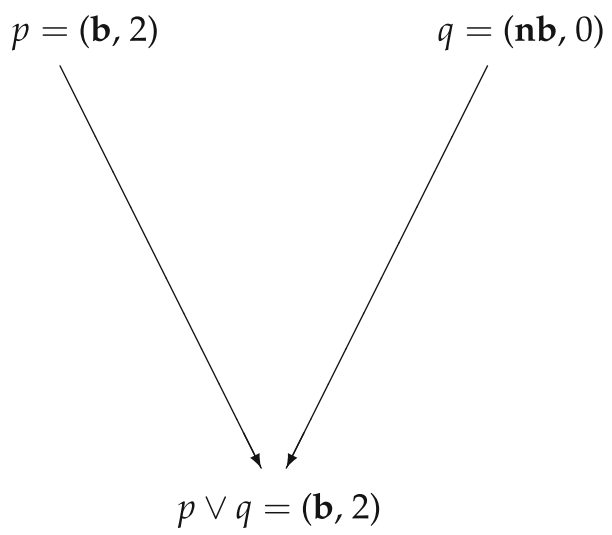

see this, consider again the case of disjunction. It is possible to rationally believe a disjunction without believing one of its disjuncts. ${ }^{18}$ One particularly simple case is the law of excluded middle: I may have no information about $p$, but I can still believe $p \vee \neg p$. In such a case, both $p$ and $\neg p$ receive the value $\mathbf{n b}$, while the disjunction receives the value $\mathbf{b}$. If the value $\mathbf{n b}$ for non-belief could be equated with falsity, then the whole disjunction should receive the value nb given that both disjuncts do. In a causal model for belief, one would implement a situation like this by treating particular instances of the law of excluded middle as basic beliefs, represented in terms of a single exogenous variable (see also Sect. 6). Note that this means that not every disjunction is believed because of the same causal mechanism. If one tried to account for a belief in $p \vee \neg p$ in terms of the structural equation associated with Fig. 2, one would find that $p \vee \neg p$ is not believed when no disjunct is believed. But this would be the wrong result for cases like the law of excluded middle. Thus, different disjunctions (and, more generally, different propositions with the same logical form) will sometimes be associated with different structural equations (I return to this point below).

Cases in which a disjunction is believed without any disjunct being believed can also occur when the disjunction is believed because of testimony. If one takes testimony to be a non-basic source of information, there will be a belief-forming mechanism generating a belief in $p$ based on testimony for $p .{ }^{19}$ Simplifying in various respects, such a mechanism might look like this:

$$
p \vee q=\left\{\begin{array}{l}
\mathbf{b} \text { if Testimony }(p \vee q)=\mathbf{b} \\
\mathbf{n b} \text { otherwise. }
\end{array}\right.
$$

\footnotetext{
18 Negation is another case in point. Although one should generally not believe $\neg p$ if one believes $p$, it is only in exceptional circumstances that one should believe $\neg p$ because one does not believe $p$. Not believing a proposition is in general not sufficient for believing its negation-oftentimes abstention of judgement is called for. For this reason, a rational belief forming mechanism for negation may assign $\mathbf{n b}$ to $\neg p$ based on $p$ being assigned $\mathbf{b}$, but it should not always assign $\mathbf{b}$ to $\neg p$ based on $p$ being assigned $\mathbf{n b}$.

19 If one takes testimony to be a basic source of information, the proposition testified to would be represented by an exogenous variable as in the case of a the law of excluded middle described above.
} 
The example of testimony also illustrates that belief-forming mechanisms are not limited to deduction. Inferring a proposition from testimony is not a deductively valid inference, even though it can transfer justification from a testimonial belief to the target proposition.

More generally, inductive and abductive inferences correspond to belief-forming mechanisms which can in principle be represented in terms of (very complex) structural equations. However, it should be pointed out that non-deductive forms of inference oftentimes do not deliver outright beliefs. The proper response to abductive or inductive arguments is oftentimes just a high credence falling short of outright belief. As causal models for doxastic justification currently just recognize outright beliefs and their absence in terms of the binary values $\mathbf{b}$ and $\mathbf{n b}$, drawing uncertain conclusions in response to non-deductive inferences is beyond their scope. But it is certainly conceivable to extend these models by adding a continuum of values, say in the unit interval, to include graded attitudes such as credences.

Let us turn now to take a closer look at structural equations in the present model for doxastic justification. The numbers assigned to them capture the epistemic strength of the belief-forming mechanism they represent. The higher the number, the stronger the epistemic link between a variable and its parents (see also Figs. 3, 4 below). How strong a given link is can depend on various factors: (i) whether the mechanism is sensitive to the right reasons in the right kind of way; (ii) how robust this sensitivity is (how easily the inference could have gone wrong); and (iii) potentially other features. For instance, not recognizing a reason as a reason for (or against) the belief in question, overestimating (or underestimating) the strength of a reason, or overlooking the presence of a defeater (think of the information that two witnesses have coordinated their testimony), all this may impact the epistemic quality of a belief-forming mechanism. ${ }^{20}$ Deviant causal chains, as discussed by Plantinga (1993: 69, fn. 8), will typically be assigned zero strength.

In this regard, it is important to emphasize that structural equations represent particular tokens of causal mechanisms. In the present model, this means that there are two structural equations for assigning values to $p \vee q$ and to $r \vee s$ respectively. Consequently, even if two such instances represent exactly the same type of reasoning, they may still be assigned different strengths. This can happen, for example, when the agent has a strong bias for or against one of the propositions in question which weakens her reasoning skills when this particular proposition is concerned. However, making room for divergences in strength between belief forming mechanisms instantiating the same type does not conflict with the plausible thought that in standard cases the strength of the tokens will be the same and can be taken to derive from a general feature of a corresponding type.

One may relate the strength of a belief-forming mechanism to the degree of doxastic justification of the output belief given various degrees of justification for the input beliefs. The idea would be this. If a belief-forming mechanism delivers a degree-1 belief as output when given degree-1 beliefs as input, but only a degree-1 belief as output when given degree- 2 beliefs as input, then its degree should just be 1 . In such

\footnotetext{
20 In this respect the present theory is less informative than those like Pollock's (1995) which attempt to make explicit the impact of defeaters on the strength of a belief-forming mechanism.
} 
Fig. 3 Structural equation $F$ assigned value 1

Fig. 4 Structural equation $F$ assigned value 2
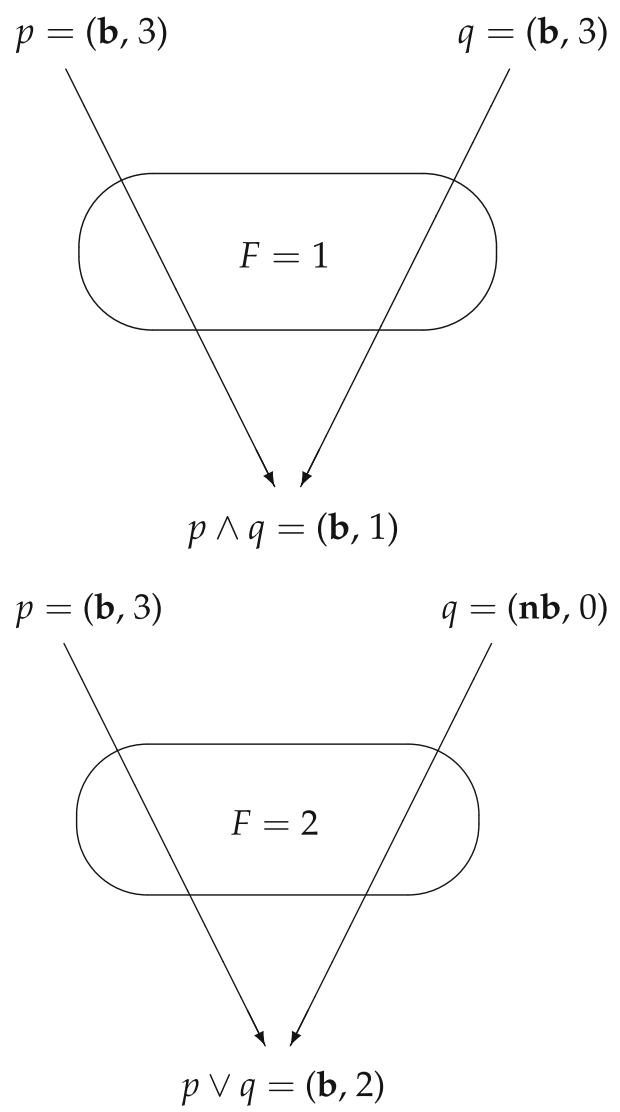

a case, the mechanism would be able to transfer a certain amount of justification, but it would not be robust or reliable enough to transfer any higher amount. Think, for instance, of a student in logic who has strong knowledge of the premises of an argument, is trained in making the relevant inference, but still not trained enough to gain, on this basis, equally strong knowledge of the conclusion. Figures 3 and 4 illustrate such situations for conjunction and disjunction (the structural equations presupposed in these examples are the one's defined on p. 7 and p. 10 respectively). In general, the strength of a belief-forming mechanism can be identified with the highest degree of justification it is able to transfer.

Here is a brief summary of where we stand. A causal model for doxastic justification determines for every proposition whether it is believed. Each endogenous variable (corresponding to a derivative belief) is associated with a structural equation describing how its value (b or $\mathbf{n b}$ ) gets determined from the variables it directly depends on (its parents, that is its immediate sub-formulas). This part of the model is purely causal: derivative beliefs are supposed to be caused by more basic beliefs as described by the structural equations (hence, base beliefs are also assumed to be temporally prior to derivative beliefs). On top of the causal part of the model, there are degrees of justification assigned to the base beliefs and degrees of strength assigned to the struc- 
tural equations. With this in place, we can now ask how these components determine degrees of justification for derivative beliefs. Before doing so, a word of clarification: regarding degrees of justification for derivative beliefs, it is best to think of them as being grounded rather than caused by the degrees of justification for the more basic beliefs and the strength of the belief forming mechanisms. According to the present picture, base beliefs cause derivative beliefs, but the degrees of justification for the latter merely supervene on the causal structure and its more basic epistemic properties.

\section{Linking Principles}

How does a derivative belief's degree of justification depend on its parent beliefs' degrees of justification? By way of a critical assessment of John Pollock's influential work on this, I defend in this section the following two constraints:

Weakest Link If a belief is based on a set of jointly sufficient reasons no proper subset of which is sufficient on its own, the degree of justification is the minimum of the degrees of these reasons. ${ }^{21}$

The Sum Rule When two or more individually sufficient reasons are independent, their degrees of justification add up.

Before discussing these constraints in detail, a few preliminary remarks are in order. The notion of a sufficient reason will be made more precise in Sect. 5. The notion of independence will ultimately only be assumed for base beliefs, which is why I will not attempt to offer a definition. As the two constraints merely serve as an informal motivation for the account to be developed, the present section presupposes only an intuitive understanding of sufficient reasons and independence. As a first pass, a set of reasons is sufficient for a belief $A$ if one believes $A$ because of these reasons and is justified in doing so. Two reasons are independent if neither is justifiably believed because of the other.

I will now discuss these constraints in turn. (To anticipate, it is no accident that both constraints are reminiscent of features of ranking theory-this parallel will be explored in Sect. 6.) For the purposes of this section, I presuppose that the beliefforming mechanisms are perfect (i.e. of strength $\infty$ ). This assumption gets relaxed in Sect. 5.3.

\subsection{Weakest Link}

Suppose one forms a belief based on two beliefs which are jointly sufficient but individually insufficient. There are no other beliefs relevant to the issue. To be precise, let us also assume the following: one of the parent beliefs has a degree of doxastic justification 1 , the other degree 2 . What could the strength of the derived belief be?

\footnotetext{
21 Talk of reasons has to be taken with a grain of salt throughout this paper. According to my usage, a reason for a belief is a justifying belief which is itself justified and causally upstream of the target belief. If justification is non-factive, then this use would be in conflict with a plausible factivity of possessed reasons. In addition, $A$ being a reason for $B$ is often used without presupposing that $A$ itself is a justified belief or that there is a causal relation between the two (Spohn 2012: Ch. 6). For more on the ideology of reasons, see Hawthorne and Magidor (2018).
} 
Pollock (2001: 244f.) suggests a straightforward answer to this question (see also Pollock 1995). ${ }^{22}$ The degree of doxastic justification of a derived belief should be the minimum of the degrees of justification it is based on. The intuitive thought behind this is that a set of reasons is only as strong as its weakest link. As observed by Pollock (2001: 244), a selling point of Weakest Link is its closure-friendliness: if all premises of an argument have a positive degree of justification (or a degree above a certain threshold), then so does a conclusion based on these premises.

Weakest Link is not uncontentious. There are reasons to think that sometimes the justification can be lower than the minimum. There are also reasons to think that it can sometimes be higher.

A reason to think that the justification can sometimes be higher is that a derivate belief can, logically speaking, be significantly weaker than the premises it is based on. For instance, one may infer $p \vee q$ from $p$. The disjunction will in general be weaker than the first disjunct. When the disjunction is weaker, isn't a belief in the disjunction more justified because there are fewer possibilities for it to be false?

In defense of Weakest Link, recall that we are dealing with doxastic justification and not merely propositional justification. What is plausible is that a weaker proposition, possibly as weak as a logical truth, enjoys more propositional justification. However, the same does not seem true of doxastic justification. If the sole reason I believe a disjunction consists in believing the first disjunct, it does not seem that I am more justified in believing the disjunction than in believing the disjunct. Of course, the situation changes if reflection on the disjunction reveals that it is significantly weaker than the disjunct. But then I have discovered a further reason to believe the disjunction. As long as the disjunct is the only reason, Weakest Link is defensible.

Let us turn now to the second challenge. It stems from the observation that risks can accumulate. If each of a number of base beliefs has a certain risk of being false, the risk involved in a derivative belief may be higher than the minimum risk. If risks are construed as probabilities, the observation comes down to the fact that a belief in a conjunction, inferred from its two conjuncts, often has a probability lower than the minimum probability of its conjuncts. ${ }^{23}$

Does this show that Weakest Link is false? This would, I think, be too hasty. What we learn is that high probability cannot be sufficient as well as necessary for a positive degree of justification. Weakest Link is plausible when one thinks of degrees of justification as scaling risk-free or safe beliefs. ${ }^{24}$ If no belief in a set of beliefs runs any risk of being false according to some standard of safety, then the above line of argument does not get off the ground: without any individual risks, there is no

\footnotetext{
22 Pollock, however, is primarily concerned with defeasible reasoning, which is not the focus of this paper.

23 As a matter of fact, the probability of a conjunction being false may be as high as the sum of the probabilities of each conjunct being false.

24 For groundwork on safety, see Pritchard (2005), Sosa (1999) and Williamson (2000: Chap. 5). A recent overview of the debate is contained in Grundmann (2018). See also the normalcy account of justification developed by Smith (2016).
} 
accumulation of risks. ${ }^{25} \mathrm{~A}$ belief based in a risk-free manner on a set of risk-free beliefs is, arguably, itself risk-free. ${ }^{26}$

It is important to note that a property like safety can still be graded (Pritchard 2014; Smith 2016: Chap. 8). A castle may be safe to degree 1 because all guards are strong enough to fend off attacks of kind 1, while they are not strong enough to fend off stronger attacks of kind 2 or higher.

However, I do not wish to identify degrees of justification with degrees of safety. A high degree of justification may merely permit the agent to treat the belief as particularly safe. ${ }^{27}$ The interpretation in terms of safety merely serves the purpose of showing that there are viable notions of degrees of justification on which something like Weakest Link can be expected to hold. Conversely, it is clear that there are weaker notions of justification, for example high probability on the evidence, for which Weakest Link fails to hold. Having a notion strong enough to satisfy Weakest Link is of interest to those who think that justified outright belief should satisfy a carefully spelled out condition of closure under competent deduction (for more on closure, see p. 31).

\subsection{The Sum Rule}

Weakest Link would not be plausible without restriction as cases of epistemic overdetermination show. To see this, suppose one has two individually sufficient reasons for a belief. Assume further that one of these reasons is stronger than the other. How strong is the derived belief's justification? It cannot be the minimum. This would mean that the resultant belief's justification is only as strong as the weaker of two sufficient reasons. But given that the second reason is sufficient as well, the justification should at least be as strong as the justification of the stronger reason. This observation led Pollock (2001: 246) to propose that in cases of epistemic overdetermination, the strength of justification corresponds to the maximum strength of the available reasons.

It seems right that if one has a sufficient reason for a belief and the belief is properly based, then the degree of justification should be at least as high as the maximal degree of justification enjoyed by the basing belief. But can't the justification sometimes be higher than the maximum (Spohn 2002) ${ }^{28}$ Suppose one has two sufficient reasons of the same strength for a belief, say of degree 1 . In a case like this, the maximum will just be 1 . So, one's justification would have the same strength that it would have had

\footnotetext{
25 Compare Smith (2016: Chap. 4) as well as Williamson's (2009) response to Hawthorne and LasonenAarnio (2009).

26 It is possible but not mandatory to connect the present line of thought to the idea that outright belief aims at knowledge (Bird 2007; Engel 2004; Smithies 2012; Williamson 2005; critically McGlynn 2013; Whiting 2013). A positive degree of justification could, on this proposal, be interpreted as potential knowledge (Jenkins Ichikawa 2014) or an approximation to knowledge (Bird 2007). On this approach, a doxastically justified belief is 'would-be-knowledge' in close or normal circumstances.

27 Degrees of justification could also be related to degrees of outright belief as explicated in Williamson (2000: 99), where degrees of outright belief grade the willingness to rely on a proposition in practical reasoning. A high degree of justification could, on this picture, be a justification strong enough to justify a high degree of outright belief in Williamson's sense.

28 See also the discussion of Pollock's approach in Spohn (2012: Chap. 11.5).
} 
Fig. 5 Two dependent reasons

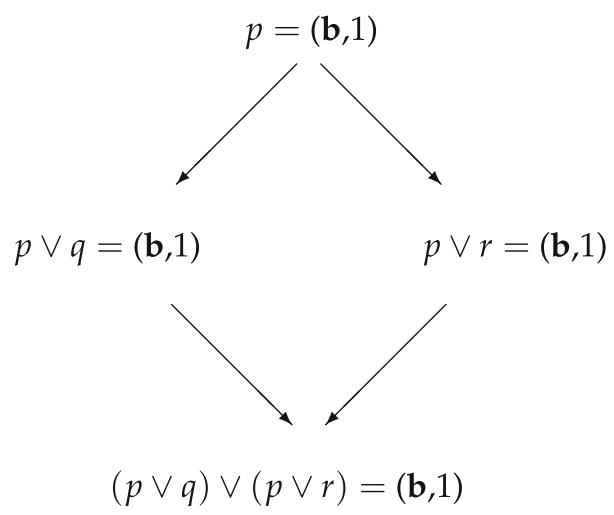

if one only possessed one of the two reasons. But more individually sufficient reasons seem to yield more justification.

Pollock (2001: 245f.) has voiced reservations about this idea. His concern is that reasons can be dependent. I may have one sufficient reason only because I have another. In such cases, it is implausible that the degrees of justification somehow add up. See Fig. 5 for illustration. In this example, the degree of a complex disjunction is not the sum of the degrees of the two disjuncts, because the two disjuncts are both believed for the same reason.

Clearly, this is a reasonable concern. The maximum-proposal appears more plausible when reasons are dependent. But when they are independent, the principle is too cautious: in such cases we can expect the degree of justification they jointly provide to be higher than the maximum (Spohn 2002). How high? A natural hypothesis would be that the degrees of independent reasons add up.

A potential weakening of the sum rule would be a rule which is merely comparative without assigning any numerical values. ${ }^{29}$ Such a comparative relation could still register many of the differences marked by the sum rule while at the same time remaining agnostic on whether degrees of justification really add up. With the sum rule in place, it makes sense to say that one's justification for a proposition $p$ is twice as high as one's justification for a proposition $q$. Although this may strike one as a strong commitment, note that we do not only compare the strength of justification but also qualify our comparisons. A justification for one proposition can not only be stronger than the justification for another, but it can also be "a lot stronger" or "barely stronger". Such qualifications can be neatly captured if the strength of justification is measured on a cardinal scale which goes beyond mere comparisons.

One can, I think, give a positive argument for the sum rule. ${ }^{30}$ First of all, it seems clear that the sum rule provides an upper bound. If a belief's degree of justification were sometimes higher than the sum, part of the belief's strength would be unaccounted for. Where would the difference come from? If a degree of doxastic justification should always be explainable in terms of the degrees of justification of the basing beliefs, it seems it should never be higher than the sum.

\footnotetext{
29 Thanks to Robert Williams for this suggestion.

30 See also Spohn (2012: Chap. 7.5) for a defense of the sum rule regarding independent testimonies.
} 
To argue that the sum rule also provides a lower bound, suppose there are three independent and sufficient reasons each enjoying a degree of justification of 1 . The sum rule would tell us that the degree of justification for a derivative belief based on these reasons is 3 . Now suppose we had a counterexample to the sum rule in this case. Then the degree of justification would be 1 or 2. First Claim: It couldn't just be 1 , for then the presence of two further independent and sufficient reasons would add nothing to the first reason. Second Claim: Degree 2 is still too low. To see this, consider a second case which results from the present case by taking one of the sufficient reasons away, so that there would only be two sufficient reasons present. The degree of justification in this second case should be 2 and not 1, for otherwise the presence of a second sufficient reason would add nothing to the overall justification. But if so, then there would be no difference between this case and the original case, even though one case comes from the other by adding another sufficient reason.

The reasoning behind this case generalizes. ${ }^{31}$ Consider a function $g(x, y)$ which takes pairs of natural numbers as input and delivers a natural number as output. Think of $g$ as a candidate function for mapping the degrees of two independent reasons on a degree for a belief supported by these reasons. Our discussion so far justifies the assumption that $g$ has the following features:

Bound. For all $x, y \in \mathbb{N}: g(x, y) \leq x+y$

Monotonicity. For all $x, y, z \in \mathbb{N}$ : If $z>y$, then $g(x, z)>g(x, y)$.

Identity. For any $x \in \mathbb{N}: g(x, 0)=x$

Bound expresses the plausible thought, illustrated above, that the support provided by two independent reasons should never be higher than the sum of their respective strengths. Monotonicity is a feature used multiple times in the preceding informal argument. It requires that when we substitute a reason with a higher degree for a reason with a lower degree, then the total support increases. Finally, Identity merely states that combining the strength of reasons is invariant under the addition of nullreasons, where a null-reason is either a proposition not believed or an unjustified belief.

It is now straightforward to observe that the only function with these properties is addition:

Observation. If a function $g$ over the natural numbers satisfies Bound, Monotonicity and Identity, then for all $x, y \in \mathbb{N}: g(x, y)=x+y$.

To see why this observation holds, consider an arbitrary natural number $x$. By Identity, $g(x, 0)=x=x+0$. Now turn to $g(x, 1)$. This value must be greater than $x$ (by Monotonicity), but not greater than $x+1$ (by Bound). But this leaves only one option: $g(x, 1)=x+1$. Iterating this argument shows $g(x, y)=x+y .^{32}$

A limitation of this argument is its restriction to the natural numbers. When the domain of potential degrees of justification is extended to the real numbers, the three

\footnotetext{
31 Thanks to an anonymous referee for pressing me on this.

32 Formally, this line of argument amounts to a proof by induction for fixed $x$ and variable $y$. Identity gives us the inductive base with $y=0: g(x, 0)=x+0$. For the inductive step, we assume that the claim holds for $y$ and show that it holds for $y+1$. By assumption, we thus have $g(x, y)=x+y$. Now we employ Monotonicity and Bound as in the main text to argue: $x+y<g(x, y+1) \leq x+y+1$, which implies $g(x, y+1)=x+y+1$ as desired.
} 
constraints no longer imply the sum rule. Further constraints would be necessary to justify the sum rule for real-valued degrees of doxastic justification.

Within these limits, the present observation provides us with a (formal) argument for the sum rule. The reasoning behind this argument indicates that without the sum rule it will be very hard to make all the distinctions we intuitively would like to make. ${ }^{33}$ One may add to this that the sum rule is simple and systematic. In particular, it has a variety of features various alternative functions lack. For instance, the sum rule is insensitive to the order of the relevant reasons (it satisfies associativity and commutativity).

In light of the foregoing considerations, I conclude that there is at least prima facie good evidence to explore the sum rule as a working hypothesis.

\section{Defining Degrees of Justification}

This section develops an account of how base beliefs pass their justification upward. The account pays close attention to the two constraints defended in the previous section. I first say something on what it could mean for a belief to be treated as a sufficient reason. Next, I consider the case of epistemic overdetermination before I incorporate the impact of possibly imperfect belief-forming mechanisms. Then, stringing it all together, a definition of a derivative belief's degree of justification is offered.

\subsection{Sufficient Reasons}

It is a truism that what one treats as a reason need not be a reason. This can happen, for instance, when believing $p$ is treated as a sufficient reason for believing $q$, while $p$ is either itself not justified or does not support $q$. The strength of the reasons one treats as sufficient can be expected to constrain the degree of doxastic justification. After all, doxastic justification is not just a matter of the strength of the reasons one possesses but also concerns the extent to which one is sensitive to these reasons.

The notion of treating a set of propositions as sufficient, which will be developed in this section, looks at base beliefs directly. This is motivated by two considerations. Firstly, the strength of a derivative belief is ultimately grounded in the strength of the base beliefs from which it is derived. Secondly, base beliefs are independent of each other (if a proposition is believed because of another belief, then it does not belong to the belief base). Focusing on base beliefs directly is superior to a recursive account which would derive the strength of derivative beliefs recursively from the strength of its parent beliefs which may depend on a common source (as Fig. 5 illustrates). ${ }^{34}$

\footnotetext{
33 To be fair, even with the sum rule in place, not all differences one intuitively would like to make could be registered. Intuitively, if one adds a further reason to one's justification which is independent but merely partial (i.e. non-sufficient), this should increase one's justification as well. But this difference will not be registered even with the sum rule in its current form. Thus the framework is coarse-grained in only reflecting different levels of sufficient justification. A possible extension of the framework could include intermediate degrees of justification, but for the time being, I must leave this for further research.

34 A recursive account could still be applicable to tree-like structures of justification in which different premise-beliefs are not justified by a common base belief. Thanks to Jonathan Schaffer for this.
} 
The envisaged notion is supposed to describe how a non-empty set of exogenous variables representing base beliefs, i.e. propositions $p_{1}, \ldots, p_{n}$, can be treated as a set of sufficient reasons for a belief in $q .{ }^{35}$ To this end, consider the following definition:

Definition 5.1 (Sufficient Reasons) A non-empty set of exogenous variables $p_{1}, \ldots, p_{n}$ are treated as sufficient for an endogenous variable $q$ in a causal model for belief if

(i) $p_{1}, \ldots, p_{n}$ as well as $q$ are assigned $\mathbf{b}$ in the model (i.e. they are believed), and

(ii) the model keeps delivering $\mathbf{b}$ as $q$ 's belief-value for all alternative contexts which change some values of exogenous variables outside of $p_{1}, \ldots, p_{n}$ from $\mathbf{b}$ to $\mathbf{n b}$.

The first condition expresses the plausible constraint that something can only be treated as a sufficient reason for a belief if it is believed itself. The second condition reflects the intuitive thought that treating something as a sufficient reason means that nothing else is required to derive the belief in question. This means that the agent would continue to hold a belief in $q$ if she were to give up beliefs other than those she treats as sufficient reasons. ${ }^{36}$ If this were not so, it would seem that the propositions in question would not be treated as sufficient after all: the belief would depend on other propositions outside of this set.

One could strengthen the present condition by requiring in addition that the causal model keeps delivering $\mathbf{b}$ when variables outside of $p_{1}, \ldots, p_{n}$ are changed from $\mathbf{n b}$ to $\mathbf{b}$. This would mean that one only treats a set of reasons as sufficient if one would continue to hold the belief on all alternative ways of adopting new beliefs. However, such a condition would be fairly strong. There is a potential defeater to almost any belief which, if adopted, would cause one to give up the belief. A set of beliefs could then only be sufficient for a belief if every potential defeater were disbelieved in the model, i.e. the defeater's negation were to receive $\mathbf{b}$.

\subsection{Epistemic Overdetermination}

The picture developed so far suggests a need to look at sets of sufficient reasons at the belief base. The degree of justification that a derivative belief enjoys could then simply be the sum of the support of a number of disjoint sets of minimally sufficient reasons. Within each such set, it makes sense to apply Weakest Link. And across different sets of sufficient reasons, it makes sense to apply the sum rule, for base beliefs are independent and the sets do not overlap. However, as I show in this section, this picture has to be qualified in an important respect. The lesson to learn is that reasons can, in a sense to be explained below, sometimes 'split their strength'.

Here is a case which proves problematic for the picture just sketched. Consider a complex conjunction, $((p \vee q) \wedge r)$, consisting of a disjunction and another proposition. Suppose that $p, q$ and $r$ are base propositions. The associated model contains just two structural equations: one which permits to infer a disjunction from its disjunct and one which permits to infer a conjunction from both conjuncts. ${ }^{37}$ Suppose further

\footnotetext{
35 Sets of reasons are assumed to be non-empty throughout.

36 McCain (2012) employs a similar clause.

37 The structural equations for conjunction and disjunction are the ones explained on p. 7 and on p. 10 respectively.
} 


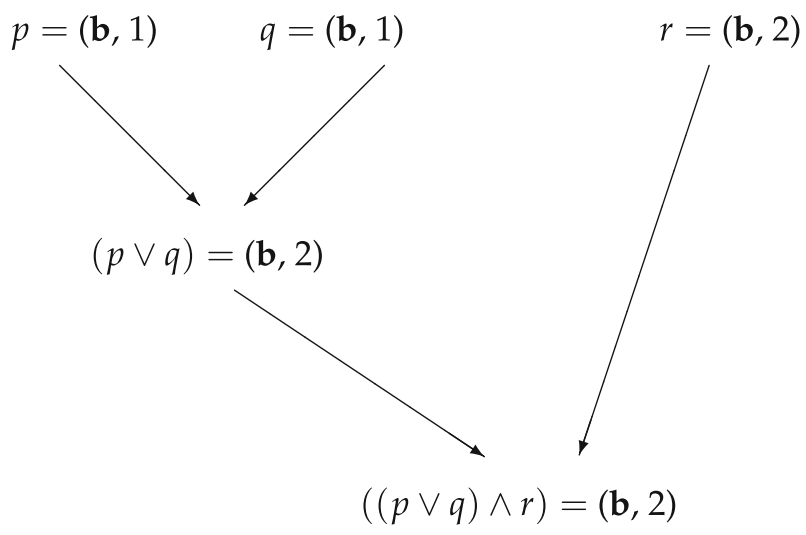

Fig. 6 Intuitive verdict about believing a conjunction

that $p$ and $q$ are both assigned degree of justification 1 (by the justification function as in Def. 3.1), while the degree of justification of $r$ is 2 . What should be the degree of justification of the complex conjunction?

Arguably, it should be 2 (as illustrated in Fig. 6). Here is why. There are actually two individually sufficient reasons for the disjunction $p \vee q$, namely $p$ and $q$, each enjoying a degree of 1 . By the sum rule, the disjunction should have degree 2 . But now observe that the conjunction is based on two reasons, which are individually insufficient but jointly sufficient: $p \vee q$ and $r$, each being assigned a degree of 2 . Given that both links are equally strong, Weakest Link implies that the conjunction should also have degree 2 , the intuitively plausible result.

However, the picture sketched at the beginning of this section returns a dissenting verdict. When looking at the base beliefs, there are only two sets which are treated as sufficient reasons for the conjunction: $\{p, r\}$ and $\{q, r\}$. Their minima are both 1 , but they are not disjoint. So we cannot find more than one non-overlapping set of sufficient reasons at the belief base and no matter how we choose, it has only degree 1 . This is the problem.

Intuitively, when we consider $\{p, r\}$ as supporting the conjunction, we ignore a further reason, namely $q$, which also supports the conjunction. But $q$ is insufficient on its own vis-à-vis the conjunction. How could one bring this reason back into play?

One way would be to consider overlapping sets of reasons, namely $\{p, r\}$ and $\{q, r\}$. In general, however, this runs the risk of overproducing justification because the support $r$ provides may be counted twice when summing over the minima of these sets. In order to balance this, reasons occurring in more than one set would have to be discounted appropriately. They would have to split their strength by contributing only some part of it when they occur in multiple sets. In the present case, this would mean that $r$, who has degree 2, would count as having just degree 1 when occurring in the two sets $\{p, r\}$ and $\{q, r\}$. Then the conjunction is indeed supported by two sets of sufficient reasons each with a minimum of 1 . And we could allow to sum over these values, because we have made sure that we do not double-count despite the fact that the sets overlap. 
To implement this idea formally, write $\left\{p, r_{1}^{1}\right\}$ and $\left\{q, r_{2}^{1}\right\}$. The lower index counts the occurrences of the proposition $r$, while the upper index states how much of its justification is contributed to the set in question. If a proposition just occurs in one set, we can leave it without indices (the lower index would be 1 and the upper index the variable's degree of justification assigned by the justification function). As a general constraint, it should be required that if $r_{1}^{i_{1}}, \ldots, r_{n}^{i_{n}}$ occur in different sets, and if $m$ is $r$ 's degree of justification, then $m=i_{1}+\cdots+i_{n}$. This is just to say that the justification that a base belief contributes to various sets of justification should ultimately sum up to its original degree.

We may capture the present idea by way of the following definition:

Definition 5.2 (Appropriate Split) Suppose $\mathcal{P}$ is a set of sets of exogenous variables (i.e. base beliefs) which are treated as sufficient for a an endogenous variable $p$ (a derivative belief; cp. Def. 5.1) in a model for doxastic justification (cp. Def. 3.2). $\mathcal{P}$ is an appropriate split relative to $p$ if

(i) it labels the occurrences of variables (i.e. base beliefs) across sets in $\mathcal{P}$, and

(ii) it assigns each labeled variable a positive number (including $\infty$ but excluding 0 ) such that the sum of these numbers for each proposition equals the proposition's degree of justification in the model.

An appropriate slit packages base beliefs into potentially overlapping sets of reasons while at the same time discounting the degrees of justification for propositions occurring in more than one set.

There are two further concepts which can be defined in terms of appropriate splits:

Definition 5.3 (Ranks) Suppose $B$ is a set of exogenous variables (i.e. base beliefs) which is an element of an appropriate split $\mathcal{P}$ (relative to some endogenous variable $p$ ). Then the rank of $B$ is the minimum of the values assigned to the elements of $B$ by $\mathcal{P}$.

Definition 5.4 (Justificatory Force) The justificatory force of an appropriate split $\mathcal{P}$ is the sum of the ranks of the sets which are elements of $\mathcal{P}$.

The rank of a set is akin to the principle Weakest Link. ${ }^{38}$ This makes sense as the sets in an appropriate split are treated as sufficient for a given proposition. However, the further proviso that no subset of these sets is treated as sufficient is not required by the definition. The purpose of this omission is simplicity: the account developed in the next section will be sensitive to this proviso without any further constraints.

The notion of justificatory force is obviously modeled after The Sum Rule. Given that overlapping sets are appropriately discounted and assuming further that base beliefs are independent, the justification they provide may be added up. Yet before making this picture more precise, we should take a look at the impact of belief-forming mechanisms.

38 It is also close to the law of minimativity in ranking theory (see Sect. 6 for more on this). 


\subsection{Imperfect Mechanisms}

In our discussion of linking principles (Sect. 4), it was assumed that the belief-forming mechanisms involved were perfect. It is now time to relax this assumption. In order to do so, recall that belief-forming mechanisms in a causal model for doxastic justification are assigned degrees of strength as well. As explained in Sect. 3, the value assigned to a belief-forming mechanism represents the highest degree of justification the mechanism is capable of transferring from its parent beliefs to the target belief. Yet when we look at the relation of a given derivative belief to the base beliefs treated as sufficient reasons, more than one mechanism may be involved. How do the values that these mechanisms have impact the belief's degree of doxastic justification?

To begin with, consider a set $B$ of base beliefs (possibly resulting from a salient split) which is treated as sufficient for a derivative belief $p$. A structural equation $F$ lies on a directed path between a base variable $v$ in this set and $q$ if there is a directed path between $v$ and $p$ so that $F$ is the structural equation associated with one of the variables on this path. A directed path between variables results from relating a variable to one of its parents, directed parent-to-offspring and chaining these links nose-to-tail. Consider all equations which lie on paths from variables in $B$ and the target variable $p$. Call these the equations involved in the justification of $p$ by $B$.

If the justificatory force of $B$ is $n$, how much justification does it confer on $p$ ? In line with the interpretation of the values assigned to belief-forming mechanisms, it suggests itself to consider the thought that $n$ is constrained by the minimum strength $m$ of the structural equations involved in the justification of $p$ by $B$. In general, the idea would be that a chain of belief-forming mechanisms starting with a number of base beliefs is as strong as its weakest link in close parallel to the thought that the strength of a set of minimally sufficient reasons is only as strong as its weakest link. ${ }^{39}$ If $m$ is not lower than $n$, then $B$ can confer its full justificatory force on $p$. However, if it is not, $p$ 's degree of justification will only be $n$. This gives rise to the following two definitions:

Definition 5.5 (Qualified Rank) Let $B$ be an element of an appropriate split $\mathcal{P}$ relative to an endogenous variable $p$. Let $m$ be the minimum strength of the belief forming mechanisms (i.e. structural equations) involved in the justification of $p$ by $B$. Then the qualified rank of $B$ is the minimum of $m$ and the rank $n$ of $B$.

Definition 5.6 (Qualified Justificatory Force) The qualified justificatory force of an appropriate split $\mathcal{P}$ relative to an endogenous variable $p$ is the sum of the qualified ranks of the elements of $\mathcal{P}$.

These two definitions attenuate the notions of rank and justificatory force by taking the strengths of the belief forming mechanisms into account. This is everything we need to finally turn to the definition of degrees of doxastic justification.

\footnotetext{
39 What if there are multiple alternative paths of justification in the model? Shouldn't such a case be parallel to cases of epistemic overdetermination, so that Weakest Link would not apply? I agree. Parallel qualifications as imposed on Weakest Link would likely be necessary. But I decided not to add any more complexity to the model at this point.
} 


\subsection{The Definition}

Here, then, finally is the definition of a belief's degree of doxastic justification.

Definition 5.7 (Degrees of Doxastic Justification) Take a causal model for doxastic justification and consider the following three cases concerning a propositional variable $p$ in the model (these cases are mutually exclusive and jointly exhaustive):

Case 1. The variable $p$ is an exogenous variable, that is a potential base belief. Then $p$ 's degree of justification is directly given by the model's justification function.

Case 2. The variable $p$ is endogenous but not believed. If this is so, then it is defined as receiving degree of justification 0 (see also p. 9).

Case 3. The variable $p$ is endogenous and believed. This entails that there is at least one appropriate split relative to $p .{ }^{40}$ Consider all appropriate splits relative to $p$. Given that our models are finite (see fn. 7), there will be only finitely many appropriate splits relative to $p$. Consider the maximum of the qualified justificatory forces of these splits. ${ }^{41}$ Define this to be $p$ 's degree of justification.

This completes the picture of degrees of doxastic justification. According to it, doxastic justification stems from the justificatory force of sets of base beliefs. It runs upwards along causal chains whose epistemic quality constrains how much justification passes through.

To see the definition in action, consider Fig. 7. A complex disjunction, $(p \wedge q) \vee r$, is believed. To what degree? With the structural equations being the obvious ones (see p. 7 and p. 10), one easily recognizes two non-overlapping sets of sufficient reasons, $\{p, q\}$ and $\{r\}$. They have ranks 1 and 3 respectively. Looking at the structural equations, one sees that $F_{1}$ does not affect these ranks, while $F_{2}$ 's degree of strength is lower than the rank of the second set. The qualified ranks are therefore 1 and 2 and their sum is 3 . It is not hard to verify that there are no splits available with a higher qualified justificatory force.

There is one feature of the definition which requires further comment. The definition takes the maximum of the (qualified) justificatory force over the available splits. One reason is that splits are not required to be complete: a given split may miss out on a further set of sufficient reasons. Besides, the sets contained in a split are not defined as minimally sufficient, although one could easily have done so. More importantly, there may be multiple ways of partitioning a belief base into sets of reasons which are treated as sufficient. The present definition reflects the thought that a belief's degree of justification corresponds to the best partition which takes all available reasons in the best possible way into account. Given that one has to deal with the possibility of multiple splits, taking the maximum is a sensible choice, for the minimum would clearly not be adequate and opting for anything in-between would, without introducing further parameters, seem arbitrary.

Degrees of doxastic justification do justice to the two constraints of Sect. 4 when applied to base beliefs (and under the assumption that the strength of the belief-forming

\footnotetext{
40 Formally, it is possible that an agent has a derivative belief without believing any base proposition. For such an odd case, the degree of justification is set to 0 .

41 In infinite models, one could consider the supremum instead of the maximum which would be allowed to include $\infty$.
} 


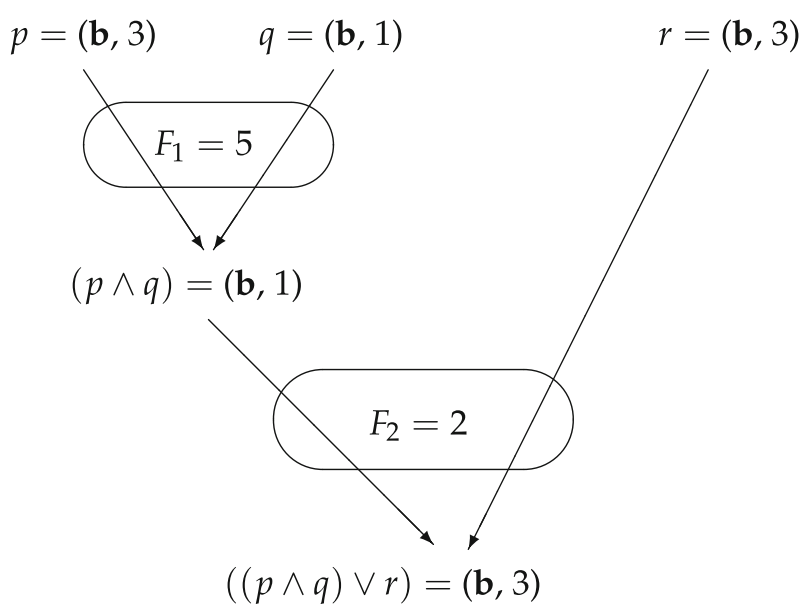

Fig. 7 The degree of justification for a complex disjunction

mechanisms is sufficiently high). First, Weakest Link holds because if there is only one set of sufficient reasons, its rank determines the degree of justification, while the rank is in turn determined by taking the minimum. Secondly, the application of the sum rule in the definition of degrees of doxastic justification ensures that sets of independent reasons add up.

\section{Perfect Thinkers}

What is the logical structure of degrees of doxastic justification? This question does not have a straightforward answer on the present account, for it permits all sorts of imperfections. Not every logical theorem must be believed. Not every logical consequence must be believed. It could even happen that the agent draws invalid inferences and therefore transfers no justification to a given conclusion. One can, however, come to a clearer view of the account's logical features by abstracting from all these imperfections. The idea would be to look at the theory's predictions for perfect thinkers. ${ }^{42}$

What characterizes a perfect thinker? As a preliminary proposal, consider the following three features:

Productivity For any (endogenous) propositional variable $p$, if the base contains a set of beliefs from which $p$ logically follows, then this set is treated as a set of sufficient reasons and $p$ is believed.

Firmness (i) For any proposition $p$, if $p$ is a logical theorem, then $p$ is believed to the maximal possible degree $(=\infty)$. (ii) All belief-forming mechanisms are assigned $\infty$ as their degree of strength.

\footnotetext{
42 Pollock (1995: Ch. 3.11) pursues a somewhat similar strategy in his discussion of ideal warrant. However, where I focus on perfect reasoning skills, his focus is on extending a given epistemic state indefinitely by successively making available all possible arguments.
} 
Correctness (i) For any proposition $p$, if a set is treated as a set of sufficient reasons, then $p$ logically follows from this set. (ii) The set of base propositions believed is consistent and each belief receives a positive degree of justification.

According to Productivity, a perfect thinker is logically omniscient. All logical consequences of her evidence are believed. Given that logical theorems are a consequence of any body of evidence, perfect thinkers also believe all logical truths. ${ }^{43}$ According to Firmness, a perfect thinker's reasoning skills are perfect. She believes logical theorems to the highest possible degree. And she may believe logical consequences of the evidence to the maximum strength that the evidence licenses. In the present framework, the latter means that the belief-forming mechanisms are working perfectly. Finally, according to Correctness, perfect thinkers make no mistakes. They do not have inconsistent beliefs and they believe a proposition only when this proposition is logically justified. It is further required that a perfect thinker only treats a set of reasons as sufficient if these reasons logically imply the belief in question. ${ }^{44}$

To offer a first idea of what follows from these constraints, let us focus on a simplified causal model for doxastic justification over a propositional language just containing (and being closed under) conjunction and negation. ${ }^{45}$ All atomic sentences are assumed to be part of the belief base, i.e. they are exogenous variables. In addition, the base may only contain logical theorems or negations of atomic sentences. Suppose further that the three constraints-Productivity, Firmness and Correctness - hold. Call any such model a model for perfect thinkers.

One can show that perfect thinkers must obey the axioms of ranking theory (a proof can be found in the "Appendix"):

Theorem In a model for perfect thinkers, the degrees of justification obey the axioms of $a$ positive ranking function.

A positive ranking function is a function $\beta$ over a propositional language into the natural numbers including 0 and $\infty$ with the following two properties (see Spohn 2012: 75):

(i) $\beta(\perp)=0$ and $\beta(\top)=\infty$, for any contradiction $\perp$ and any logical theorem $\top$. Moreover, $\beta(\phi)=\beta(\psi)$ if $\phi$ and $\psi$ are logically equivalent. ${ }^{46}$

(ii) $\beta(\phi \wedge \psi)=\min \{\beta(\phi), \beta(\psi)\}$, for all sentences $\phi$ and $\psi$ in the language.

Ranking theory shares with probability theory the feature that logical theorems and logical contradictions are assigned the top and bottom element of their respective range of values. The crucial property of ranking theory is its second axiom stating

\footnotetext{
43 Perfect thinkers are not required to be opinionated about everything. They are allowed to be agnostic about non-logical matters. Formally, this is possible because base propositions may receive degree of justification 0 .

44 This last feature is likely to be an over-simplification, yet without it one would face the difficult task of stating in formally tractable but non-logical terms what it means to respond correctly to a given set of reasons.

45 I consider sentences instead of propositions in order to have a clear syntactic structure.

46 If ranks are defined over an algebra of possible worlds, logically equivalent propositions are identified and thus automatically receive the same rank. But given that the present definition targets sentences, an additional requirement is necessary.
} 
that the rank of a conjunction is the minimum rank of its conjuncts. This axiom is reminiscent of Weakest Link, for a conjunction is assigned the weakest value out of the values of its canonical reasons, its conjuncts. ${ }^{47}$

The theory of degrees of justification presented in this paper is non-ideal. But when applied under ideal conditions, it delivers a plausible theory of degrees of doxastic justification: ranking theory. ${ }^{48}$ Spohn $(2012: 481)$ argues extensively in favor of this connection: "Degrees of justification or justifiedness are degrees of (justified, rational) belief and hence positive ranks." 49 One major reason for orienting degrees of doxastic justification towards ranking theory is the closure-friendliness of ranking theory. For any positive threshold $z$, the set of propositions with rank $z$ or higher is closed under logical consequence (see also the discussion of Weakest Link in Sect. 4.1). This guarantees that under ideal conditions, a notion of (doxastically) justified outright belief defined in terms of some such threshold $z$ is closed under logical consequence, even when this threshold is non-maximal (it can be as low as $z=1$ ). Thus, one's beliefs can be justified and closed without the required strength of justification being so high as to invite skepticism. Compare this with a probabilistic theory of justification, where the only positive such threshold is the maximal value of probability 1 . In turn, one has to work much harder to square a plausible closure of justified outright belief under competent deduction with a gradable notion of justificatory strength. ${ }^{50}$

\section{Conclusion}

The model of doxastic justification presented herein describes how base beliefs pass their justification upward along causal chains. The resulting theory of doxastic justification is closure-friendly: it can account for the fact that doxastic justification may be preserved under competent deduction. It is also fairly realistic: it makes no assumptions regarding logical omniscience or the like. Moreover, the theory can be idealized: when applied to perfect thinkers, it converges to ranking theory. The account can therefore be seen as a 'doxastified' version of ranking theory.

There are a number of further issues. The literature on causation in causal models supplies a rich assembly of dependency relations, many of which would be candidates

\footnotetext{
47 See also Spohn (2002) on the similarities between ranking theory and Pollock's theory.

48 Still open is the question what would happen if some of the constraints on perfect thinkers were dropped (do other known plausibility measures — in the sense of Friedman and Halpern (2013)_arise?) and, more generally, which rationality postulates for perfect thinkers correspond to which properties of degrees of justification.

49 See also Smith (2016: Chap. 8) for a connection between ranks and degrees of (propositional) justification.

50 In the debate about degrees of belief, Leitgeb (2014) and others observe that sets of propositions can be closed even when they include, or even primarily consist of, propositions with probability less than 1. However, this is only possible by sometimes excluding propositions from the belief set which have a probability no less than those contained in it (there is no getting around the fact that satisfying a probabilistic threshold less than 1 does not always result in a closed set of propositions). Leitgeb achieves this restriction by way of a form of contextualism about belief, so that propositions which may get in the way of closure do not count as being believed in contexts in which the threshold is comparatively low. As far as I can see, it might well be possible to extend Leitgeb's theory to a theory of degrees of doxastic justification. The prize to pay would be a very specific form of contextualism about belief.
} 
to be tried in the present model. In addition, it would be informative to see how the model can be applied to the debate about transmission failures (Wright 2002). There are also interesting structural parallels to the debate about grounding, specifically Schaffer's (2016) account of grounding in terms of causal models. A further question would be whether credal states could be added to the model, and if so, how. It would also be good to know whether the model can be made dynamic — what happens if the agent discovers new, possibly undermining evidence? Alas, these are questions for another day.

Acknowledgements I would like to thank the members of the research group "Knowledge and Decision"Roman Heil, Jakob Koscholke, Patricia Rich-for extremely helpful comments and suggestions at more than one occasion. Moreover, I would like to thank the organizers and participants of events in Barcelona, Bochum (special thanks to Catrin Campbell-Moore for extensive comments!) and Hamburg for providing me with the opportunity to present and discuss earlier versions of this paper. There are also two anonymous referees of this journal to thank for extremely detailed comments, which are much appreciated. Research for this paper has profited from the generous support of the Deutsche Forschungsgemeinschaft (DFG) under research Grant SCHU 3080/3-1/2/3.

Funding Open Access funding enabled and organized by Projekt DEAL.

Open Access This article is licensed under a Creative Commons Attribution 4.0 International License, which permits use, sharing, adaptation, distribution and reproduction in any medium or format, as long as you give appropriate credit to the original author(s) and the source, provide a link to the Creative Commons licence, and indicate if changes were made. The images or other third party material in this article are included in the article's Creative Commons licence, unless indicated otherwise in a credit line to the material. If material is not included in the article's Creative Commons licence and your intended use is not permitted by statutory regulation or exceeds the permitted use, you will need to obtain permission directly from the copyright holder. To view a copy of this licence, visit http://creativecommons.org/licenses/by/4.0/.

\section{Appendix}

This "Appendix" provides a proof of the theorem in Sect. 6. To recall, the theorem is:

Theorem In a model for perfect thinkers, the degrees of justification obey the axioms of $a$ positive ranking function.

Proof I split the proof into two parts according to the two defining properties of a ranking function. Let $\beta$ be an assignment of degrees of doxastic justification according to the definition developed in Sect. 5.4.

$i$. Firstly, note that the range of a ranking function, $\mathbb{N}_{0}^{\infty}$, is identical to the range of degrees of doxastic justification. Secondly, the demand $\beta(\top)=\infty$, where $\top$ may be any logical theorem, is satisfied by definition (Firmness (i)). What remains to be shown is $\beta(\perp)=0$ : any contradiction gets degree of justification 0 . To this end, suppose $\phi$ is a logical contradiction. It is clear that $\phi$ cannot be part of the base, because the base beliefs are consistent in the light of Correctness (ii). Therefore, $\phi$ must be an endogenous variable. For reductio, suppose $\phi$ receives a positive degree of justification. From this it follows that it is believed as well, for only beliefs receive positive degrees. For obvious reasons, the set of all base beliefs is treated as a set of sufficient reasons for $\phi$. By Correctness (i), a contradiction would follow from a consistent set. 
It remains to be shown that logically equivalent propositions receive the same degree of doxastic justification. To this end, consider two propositional variables $\phi$ and $\psi$ which are logically equivalent. Let us distinguish three cases. First, assume $\phi$ and $\psi$ are part of the belief base. Recall that in a model for perfect thinkers, the belief base may only contain atomic sentences, negations of atomic sentences, and logical theorems. As no two atomic sentences or negations thereof are logically equivalent, $\phi$ and $\psi$ must be logical theorems. As such, they both receive degree $\infty$. Second, assume that neither $\phi$ nor $\psi$ are part of the belief base, i.e. they are both endogenous variables. Observe that any two logically equivalent sentences are implied by exactly the same set of sentences. Hence, a set $B$ in the belief base implies $\phi$ iff it implies $\psi$. Correctness ensures further that a set is treated as sufficient for $\phi$ iff it is treated as sufficient for $\psi$. From this it follows that something is an appropriate split relative to $\phi$ iff it is an appropriate split relative to $\psi$. But this means that they will receive the same degree of justification. Third, suppose without loss of generality that $\phi$ is part of the belief base while $\psi$ is not. If both are logical theorems, they are already guaranteed to receive the same degree of justification. If they are not, this means that neither is a logical theorem. But then $\phi$ is actually an atomic sentence or a negation of an atomic sentence. Now given that $\phi$ is logically sufficient for $\psi$, it alone forms a set of sufficient reasons. This implies $\beta(\phi) \leq \beta(\psi)$. To see the converse, note that any set logically sufficient for $\psi$ must also be logically sufficient for $\phi$. But any consistent set logically sufficient for an atomic sentence or its negation must contain this sentence or its negation. This shows that any set in a appropriate split for $\psi$ must contain $\phi$. Given that qualified ranks of sets go with the minimum, they cannot sum up to more than the degree of justification for $\phi$, which is all we wanted to see.

ii. The second defining property of a ranking function is this: $\beta(\phi \wedge \psi)=$ $\min \{\beta(\phi), \beta(\psi)\}$, for all sentences $\phi$ and $\psi$ in the language.

We shall start by focusing on a special case (to be generalized below). Suppose (a) the conjunction is a derivative belief state, (b) both conjuncts are derivative belief states, (c) none of these three is a logical theorem, and (d) all receive a positive degree of justification. One first shows: $\beta(\phi \wedge \psi) \leq \min \{\beta(\phi), \beta(\psi)\}$.

Given assumptions (a)-(d), there is a (non-empty) set of sets treated as sufficient reasons such that the degree of justification for the conjunction is the sum of the minima of these sets. This simplification is possible because belief-forming mechanisms receive degree $\infty$ (Firmness (ii)).

Given Correctness (i), the conjunction follows from these sets. But then the individual conjuncts follow from these sets as well. By Productivity, these sets are treated as sufficient reasons for the individual conjuncts. So, there is at least one appropriate split according to which the degree of justification of the individual conjuncts is as high as the conjunction, showing $\beta(\phi \wedge \psi) \leq \min \{\beta(\phi), \beta(\psi)\}$.

To show the converse, $\beta(\phi \wedge \psi) \geq \min \{\beta(\phi), \beta(\psi)\}$, take for each conjunct a set of sets of reasons treated as minimally sufficient. Again, by Correctness (i), they will imply the conjunct for which they are treated as sufficient. Suppose, without loss of generality, that $\beta(\phi)$ equals $\min \{\beta(\phi), \beta(\psi)\}$. Then we need to show that we can construct a set of sets of sufficient reasons for the conjunction.

If any of the minimally sufficient sets has a minimum $n$ greater than 1 , then we can duplicate the set $n$ times, so that each such set has a minimum of 1 . How? Recall that 
sets of reasons may overlap (Sect. 5.2). So, if the minimum of a set of reasons is $n$, this means that all members have a degree of justification $\geq n$. Therefore, we can divide the strength of each element $n$ times while preserving a positive degree of justification (the degree of justification is 1 each time for the element with minimum degree). This way, all elements split their strength on $n$ sets whose minima are all 1 .

Apply this procedure to all sets of sufficient reasons for both conjuncts. What we find is that there is a set of sets of minimally sufficient reasons which all have minimum degree 1 . Given that $\beta(\phi)$ is the minimum, the number of sets for $\phi$ is not higher than the number of sets for $\psi$. So we can merge each set of sufficient reasons for $\phi$ with a set of sufficient reasons for $\psi$. The number of sets so achieved will be the number of sets for $\phi$ and each set will have as its minimum 1. Given that each such set contains reasons logically sufficient for $\phi$ and reasons logically sufficient for $\psi$, it contains logically sufficient reasons for their conjunction. By Productivity, these sets are treated as sufficient reasons. And given that their number is the degree of justification $\phi$ enjoys, which was assumed to be the minimum of $\{\beta(\phi), \beta(\psi)\}$, the degree of justification of the conjunction will be at least as high as the sum of these set of sufficient sets. This shows $\beta(\phi \wedge \psi) \geq \min \{\beta(\phi), \beta(\psi)\}$ and completes the first part of the proof. What remains to be done is to relax assumptions (a)-(d).

If the conjunction is a theorem, then both conjuncts are theorems. As any theorem receives degree $\infty$ in the model, feature (ii) is satisfied. When the conjunction is not a theorem, then at most one of the two conjuncts can be a logical theorem. Moreover, in such a case, the conjunct which is not a theorem will be logically equivalent to the conjunction. With this in mind, it is easy to see how to adjust the main proof above to cover this case.

Now consider a case when at least one of the two conjuncts is a base belief. By definition of a model for perfect thinkers, the conjunction can only be part of the base if it is a logical theorem and this case has already been dealt with. So we can assume that the conjunction is a derivative belief state and at least one of the conjuncts is a base belief. Again, it is not hard to see how to adjust the main proof above: instead of sets of sufficient reasons for the individual conjunct, one has to consider the conjunct itself when it is part of the base. One observation proves helpful in this regard: there cannot be sufficient reasons in the base except for the base belief itself as long as it is not a logical theorem. As observed earlier, no combination of (consistent) atomic sentences (or negations thereof) implies another atomic sentence (or its negation).

Finally, we also have to consider the case where either the conjunction or one of the conjuncts receives degree of justification 0 . If the conjunction has degree 0 , then there cannot be logically sufficient reasons in the base. But then there can neither be logically sufficient reasons for both conjuncts in the base, and so at least one of the two conjuncts receives degree 0 as well. Conversely, if one of the conjuncts receives degree 0 , then there are no logically sufficient reasons for it in the base (or, if it is part of the base, it is not believed). But then there cannot be logically sufficient reasons in the base for the conjunction either. 


\section{References}

Alston, W. P. (1976). Two types of foundationalism. The Journal of Philosophy, 73, 165-85.

Audi, R. (1983). The causal structure of indirect justification. The Journal of Philosophy, 80, 398-415.

Berker, S. (2015). Coherentism via graphs. Philosophical Issues, 25, 322-52.

Bird, A. (2007). Justified judging. Philosophy and Phenomenological Research, LXXIV, 81-110.

Bonjour, L. (1976). The coherence theory of empirical knowledge. Philosophical Studies, 30, 281-312.

Cohen, S. (1999). Contextualism, skepticism, and the structure of reasons. Philosophical Perspectives, Epistemology, 13, 57-89.

Egan, A. (2007). Epistemic modals, relativism and assertion. Philosophical Studies, 133, 1-22.

Engel, P. (2004). Truth and the aim of belief. In D. Gillies (Ed.), Laws and models in science (pp. 77-97). London: King's College Publications.

Fantl, J., \& McGrath, M. (2002). Evidence, pragmatics, and justification. The Philosophical Review, CXI, 67-94.

Feldman, R., \& Conee, E. (1985). Evidentialism. Philosophical Studies, 48, 15-34.

Friedman, N., \& Halpern, J. (2013). Plausibility measures: A user's guide. In Proceedings of the eleventh conference on uncertainty in AI.

Gerken, M. (2011). Warrant and action. Synthese, 178, 539-547.

Greenough, P., \& Pritchard, D. (Eds.). (2009). Williamson on knowledge. Oxford: Oxford University Press. Grundmann, T. (2018). Saving safety from counterexamples. Synthese, published as online first.

Halpern, J. Y., \& Hitchcock, C. (2010). Actual causation and the art of modeling. In R. Dechter, H. Geffner, \& J. Halpern (Eds.), Heuristics, probability and causality: A tribute to Judea Pearl (pp. 383-406). Norcross: College Publications.

Hansson, S. O. (1999). Taking belief bases seriously. In D. Prawitz \& D. Westerståhl (Eds.), Logic and philosophy in Uppsala, synthese library (Vol. 236, pp. 13-28). Dordrecht: Kluwer Academic Publishers.

Hawthorne, J. (2005). The case for closure. In M. Steup \& E. Sosa (Eds.), Contemporary debates in epistemology (pp. 26-43). Oxford: Blackwell.

Hawthorne, J., \& Lasonen-Aarnio, M. (2009). Knowledge and objective chance. Greenough and Pritchard, 2009, 92-108.

Hawthorne, J., \& Magidor, O. (2018). Reflections on the ideology of reasons. In D. Starr (Ed.), Oxford handbook of reasons and normativity. Oxford: Oxford University Press. chap. 5.

Hawthorne, J., \& Stanley, J. (2008). Knowledge and action. The Journal of Philosophy, 105, 571-90.

Jenkins Ichikawa, J. (2014). Justification is potential knowledge. Canadian Journal of Philosophy, 44, 184-206.

Kiesewetter, B. (2017). The normativity of rationality. Oxford: Oxford University Press.

Korez, K. A. (1997). Recent work on the basing relation. American Philosophical Quarterly, 34, 171-91.

Leitgeb, H. (2014). The stability theory of belief. Philosophical Review, 123, 131-71.

Lord, E. (2018). The importance of being rational. Oxford: Oxford University Press.

McCain, K. (2012). The interventionist account of causation and the basing relation. Philosophical Studies, $159,357-82$.

McGlynn, A. (2013). Believing things unknown. Nô̂s, 47, 385-407.

Neta, R. (2019). The basing relation. Philosophical Review, 128, 179-217.

Pearl, J. (2000). Causality: Models, reasoning, and inference. New York: Cambridge University Press.

Plantinga, A. (1993). Warrant: The current debate. Oxford: Oxford University Press.

Pollock, J. L. (1995). Cognitive carpentry. Cambridge, MA: MIT Press.

Pollock, J. L. (2001). Defeasable reasoning with variable degrees of justification. Artificial Intelligence, $133,233-82$.

Pritchard, D. (2005). Epistemic luck. New York: Oxford University Press.

Pritchard, D. (2014). The modal account of luck. Metaphilosophy, 45, 594-619.

Rott, H. (2000). Just because: Taking belief bases seriously. In S. Buss, P. Hájek, \& P. Pudlák (Eds.), Logic colloquium '98, lecture notes in logic (Vol. 13, pp. 387-408). Urbana: Association for Symbolic Logic.

Schaffer, J. (2016). Grounding in the image of causation. Philosophical Studies, 173, 49-100.

Smith, M. (2016). Between probability and certainty. Oxford: Oxford University Press.

Smithies, D. (2012). The normative role of knowledge. Noûs, 46, 265-88.

Sosa, E. (1999). How to defeat opposition to Moore. Noûs, 33, 141-53.

Spohn, W. (2002). A brief comparison of Pollock's defeasible reasoning and ranking functions. Synthese, 131, 39-56. 
Spohn, W. (2012). The laws of belief. Oxford: Oxford University Press.

Stanley, J. (2005). Knowledge and practical interests. Oxford: Oxford University Press.

Weatherson, B. (2012). Knowledge, bets and interests. In Knowledge ascriptions (pp. 75-105). Oxford: Oxford University Press.

Whiting, D. (2013). Nothing but the truth: On the norms and aims of belief. In T. Chan (Ed.), The aim of belief (pp. 184-203). Oxford: Oxford University Press.

Williamson, T. (2000). Knowledge and its limits. Oxford: Oxford University Press.

Williamson, T. (2005). Knowledge, context and the agent's point of view. In G. Preyer \& G. Peter (Eds.), Contextualism in philosophy (pp. 91-114). Oxford: Oxford University Press.

Williamson, T. (2009). Replies to critics. Greenough and Pritchard, 2009, 279-384.

Wright, C. (2002). (Anti-)sceptics simple and subtle: G. E. Moore and John McDowell. Philosophy and Phenomenological Research, 65, 330-48.

Publisher's Note Springer Nature remains neutral with regard to jurisdictional claims in published maps and institutional affiliations. 